\title{
Rapporteur Talk: Solar and Heliospheric
}

\author{
Du Toit Strauss ${ }^{a, *}$ \\ ${ }^{a}$ Center for Space Research, \\ North-West University, \\ 10 Hoffmanstreet, Potchefstroom, South Africa
}

E-mail: dutoit.strauss@nwu.ac.za

This rapporteur paper summarizes the 118 contributed presentations on solar and heliospheric physics (i.e. the $\mathrm{SH}$ sessions) presented at the $37^{\text {th }}$ International Cosmic Ray Conference. These presentations discussed various aspects of cosmic ray acceleration, transport, and modulation in the heliosphere. New and novel cosmic ray measurement techniques and related instrumentation were also presented.

$37^{\text {th }}$ International Cosmic Ray Conference (ICRC 2021)

July 12th - 23rd, 2021

Online - Berlin, Germany

\footnotetext{
${ }^{*}$ Presenter
} 


\section{The first virtual ICRC}

The solar and heliospheric physics (SH) sessions at the $37^{\text {th }}$ International Cosmic Ray Conference (ICRC) featured 118 contributions, spread over various topics regarding cosmic ray acceleration, transport, and measurements in the heliosphere. In this review paper, I will attempt to succinctly summarize the main results that caught my eye. Of course, I am biased by my own research interests and I apologize in advance for not begin able to showcase all the excellent work presented at the conference.

Due to the COVID-19 pandemic, the 2021 ICRC was moved to a virtual setting that changed the traditional oral $v s$ poster format of previous ICRCs: The traditional oral sessions were replaced with a number of discussion sessions, featuring a focused science topic to be discussed and managed by session conveners, and an informal forum meeting space to discuss results of the traditional poster presentations.

As part of the SH-session science, two review talks (by Pesce-Rollins and Malandraki) and three highlight talks (by Rankin, Berdugo, and Crosby) were also presented at the conferences. The presentations are not included in the present discussions.

In my summary below, I divide the science topics into the different discussion sessions and discuss these separately.

\section{Cosmic rays in the heliosphere}

Fig. 1 illustrates the different cosmic ray (CR) species found inside the heliosphere. From the Sun, moving outwards, these are:

- The solar energetic particle (SEP) component, being accelerated close to the Sun during transient solar phenomena. The dominant acceleration processes include magnetic reconnection in solar flares and diffusive shock acceleration occurring at the shocks driven by coronal mass ejections.

- Low energy Jovian electrons, accelerated and released from the Jovian magnetosphere.

- The anomalous cosmic ray (ACR) component, believed to be accelerated through diffusive shock acceleration at the heliospheric termination shock (TS; located at approximately 90AU from the Sun).

- The galactic cosmic ray (GCR) component that enters the heliosphere by crossing the heliopause (HP; located at approximately 120AU in the upwind direction) after being accelerated at astrophysical systems (e.g. supernova remnants) and propagating diffusively through the galaxy.

Also illustrated on Fig. 1 is a selection of the fleet of spacecraft measuring CR intensities in-situ in the heliosphere. Most notably, and especially relevant for this conference, are: The Parker Solar Probe (PSP) and Solar Orbiter (SolO) spacecraft, currently exploring the innermost regions of the heliosphere, and the Voyager 1 (V1) and 2 (V2) spacecraft exploring the interstellar medium. At Earth, AMS-02, installed on the international space stations, provides very high precision GCR 


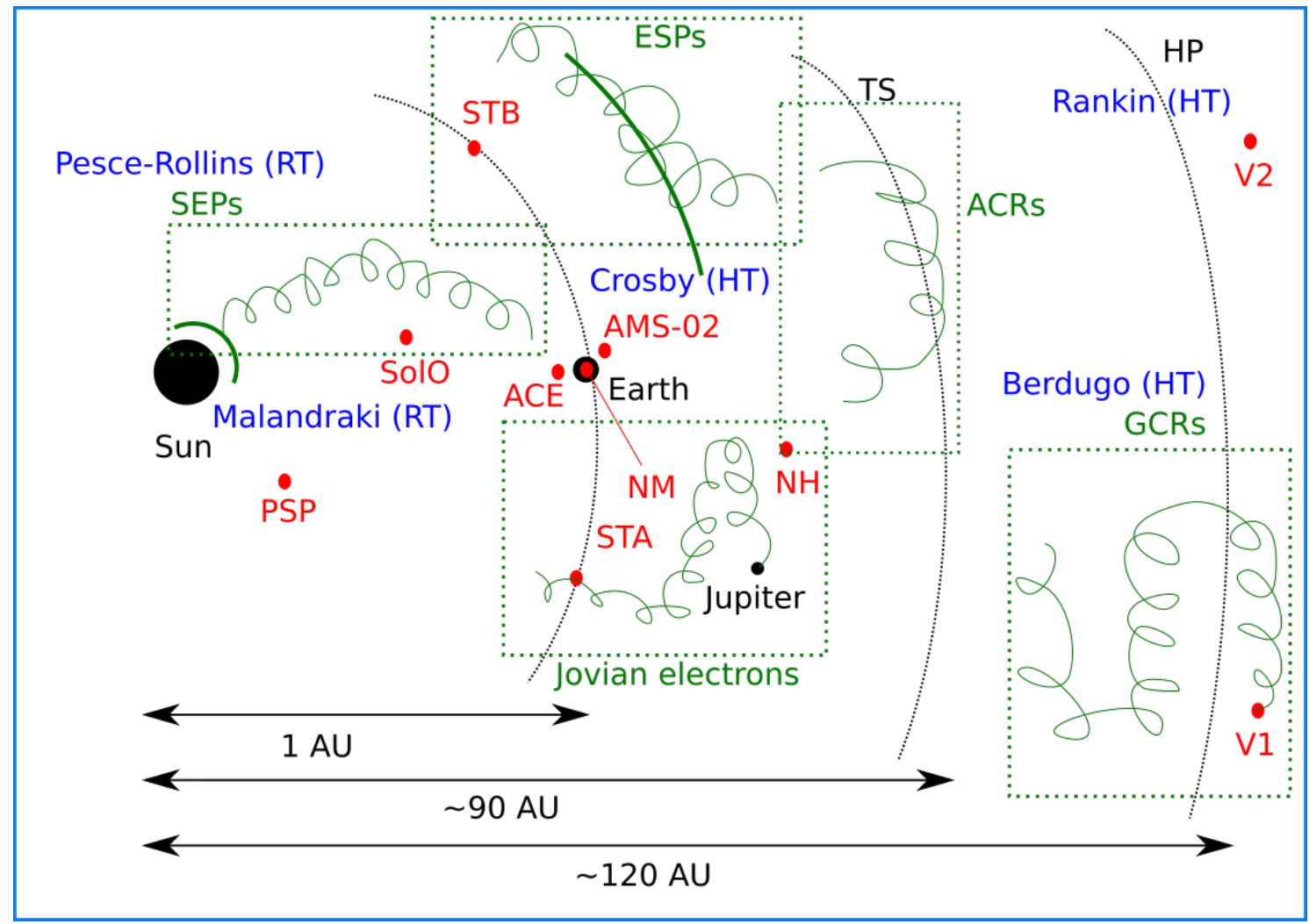

Figure 1: The figure, which is not to scale, illustrates the various CR species found in the heliosphere. Topics covered during the review (RTs) and highlight talks (HTs) are broadly indicated.

measurements, while the neutron monitor (NM) network, located on Earth surface, provides a very long-term data set ( 70 years) of indirect GCR measurements.

Such long-term GCR observations are shown in Fig. 2. These measurements, by the South African NM network, are normalised to $100 \%$ in March 1987. The previous 2009 solar minimum (in a $A<0$ drift cycle) was dubbed as being "unusual" due to the record-setting GCR intensity observed. The current 2020 solar minimum was equally quiet, with very low levels of solar activity. Due to the present $A>0$ drift cycle, GCR intensities at low energies were expected to be even higher [1] and this was indeed observed [2]. At higher NM energies, the 2020 intensities are slightly lower than those of 2009 (as expected from drift considerations), while still being considerable higher than that of any previous $A>0$ minimum. This indicates that the "unusually" quiet solar modulations conditions again prevailed in this current 2020 solar minimum. While such quiet solar conditions are not ideal for studying transient solar phenomena (e.g. SEP events), they do present the ideal conditions to study GCR modulation and many such results are summarized in this paper.

\section{Discussion session \#19: SEP acceleration and transport}

Solar energetic particles (SEPs) are accelerated, very close to the Sun, during coronal mass ejections (CMEs) and solar flares. The dominant acceleration process is still contentious and a twosource paradigm has been established: So-called gradual (i.e. proton rich) events are associated 


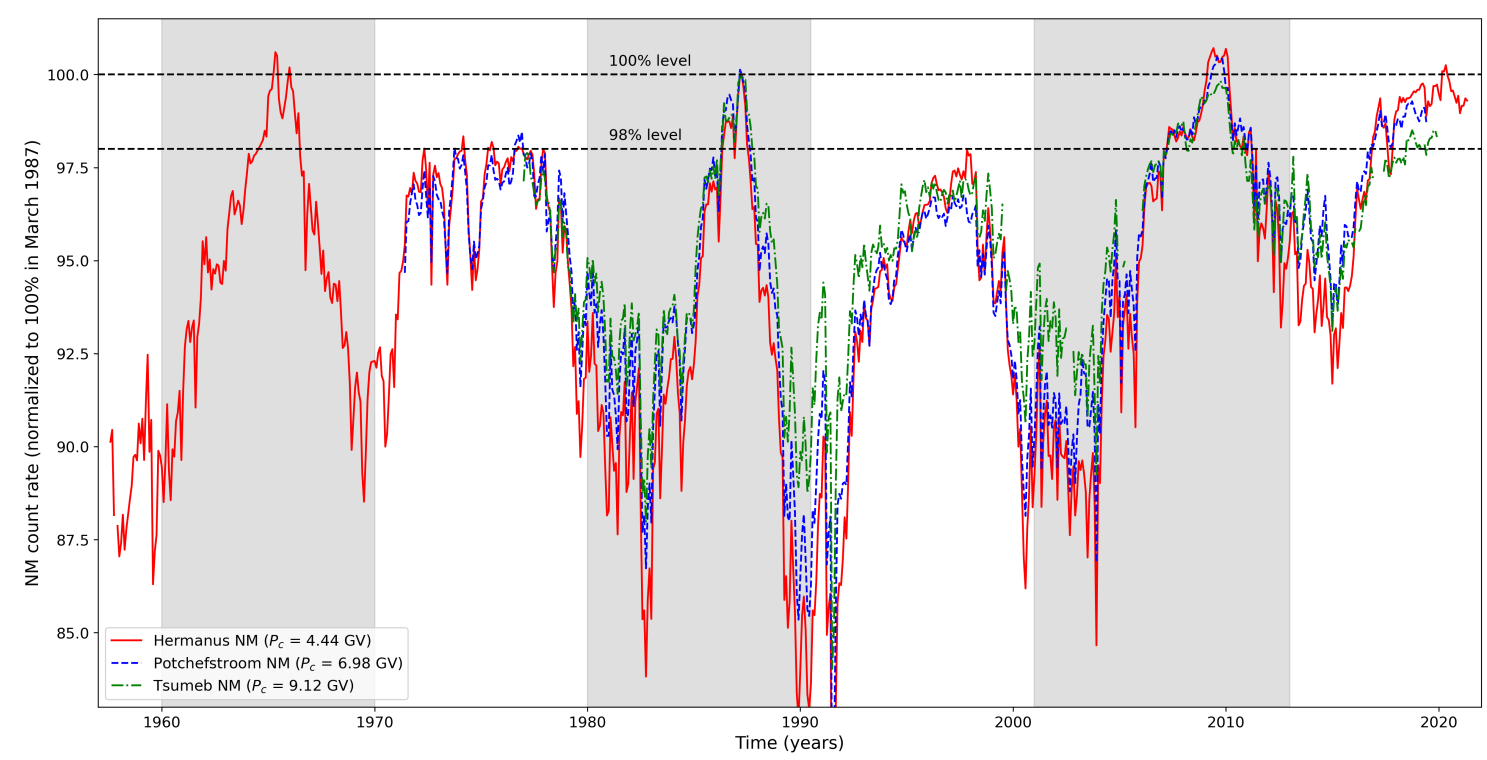

Figure 2: Long-term NM measurements, from http://natural-sciences.nwu.ac.za/ neutron-monitor-data, as recorded by the South African NM network.

with diffusive shock acceleration at CMEs, while so-called impulsive (i.e. electron rich) events are associated with acceleration via magnetic reconnection in solar flares. To disentangle the different signatures of different acceleration mechanisms, SEP observations closer to the acceleration source is needed, and this can, in future, be provided by the Parker Solar Probe (PSP) spacecraft. Fig. 3 shows PSP measurements of the energy spectra, for several species, during the 29 November 2020 SEP event, which was the first mid-sized SEP events observed by PSP after the very quiet solar minimum. We are looking forward to more such observations from PSP at it gets closer to the Sun and solar activity starts to pick up.

Before SEPs can be observed in-situ, they have to traverse the turbulent heliospheric magnetic field between the source and observer. Transport through this field can modulate the accelerated spectrum, making it very difficult to disentangle acceleration and transport effects. It is usually assumed that particles undergo sufficiently efficient scattering so that their transport can be described as a diffusive process, where $(\Delta x)^{2} \propto \Delta t$, i.e. the increments of their motion, $\Delta x$, when squared, is linearly related to the time increment $\Delta t$. However, as pointed out by [5] there is some evidence that this is not always the case in the heliosphere and, under certain conditions, we could have anomalous transport, characterized by $(\Delta x)^{2} \propto(\Delta t)^{\zeta}$, where $1<\zeta<2$ corresponds to superdiffusion and $0<\zeta<1$ to subdiffusion. The nature of the transport process has important implications for our currently used transport models, and these may have to be adapted to model non-diffusive transport.

A complete description of the transport of charged particles through a turbulent plasma is therefore still lacking. This is a fundamental problem when modelling SEP transport: The transport (or diffusion) coefficients that couple the particle motion to the underlying magnetic turbulence (e.g. the pitch-angle diffusion coefficient) are not completely known. And without knowing these coefficients, it is difficult to make robust statements and predictions regarding particle transport. This is illustrated by [6], with their results shown in the right panel of Fig. 4, where these authors 

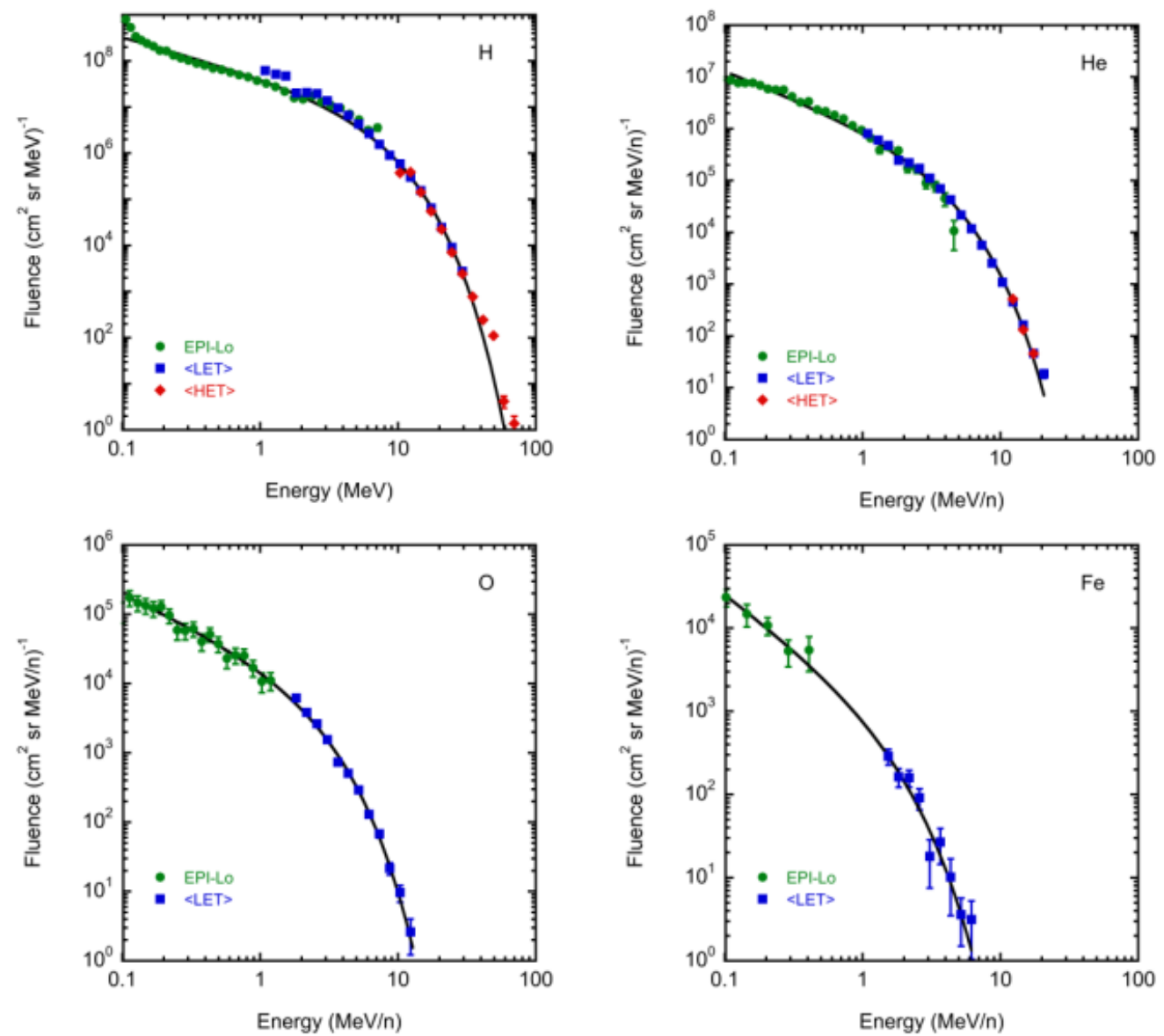

Figure 3: Parker Solar Probe fluence spectra of the 29 November 2020 SEP event. The figure is taken from [4].
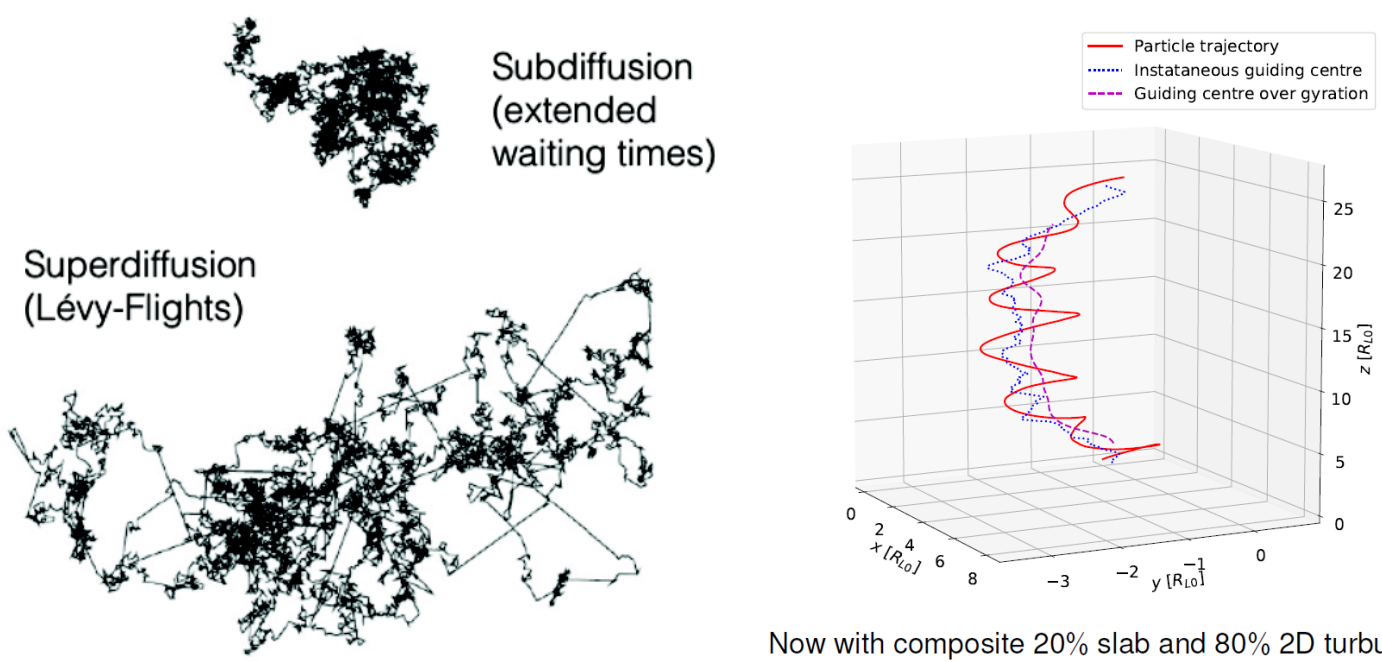

Now with composite $20 \%$ slab and $80 \% 2$ D turbulence

Figure 4: The left panel, from [5], shows a representation of a particle undergoing anomalous diffusion. The right panel, from [6], shows the trajectory of a charged particle propagating through a turbulent plasma. 

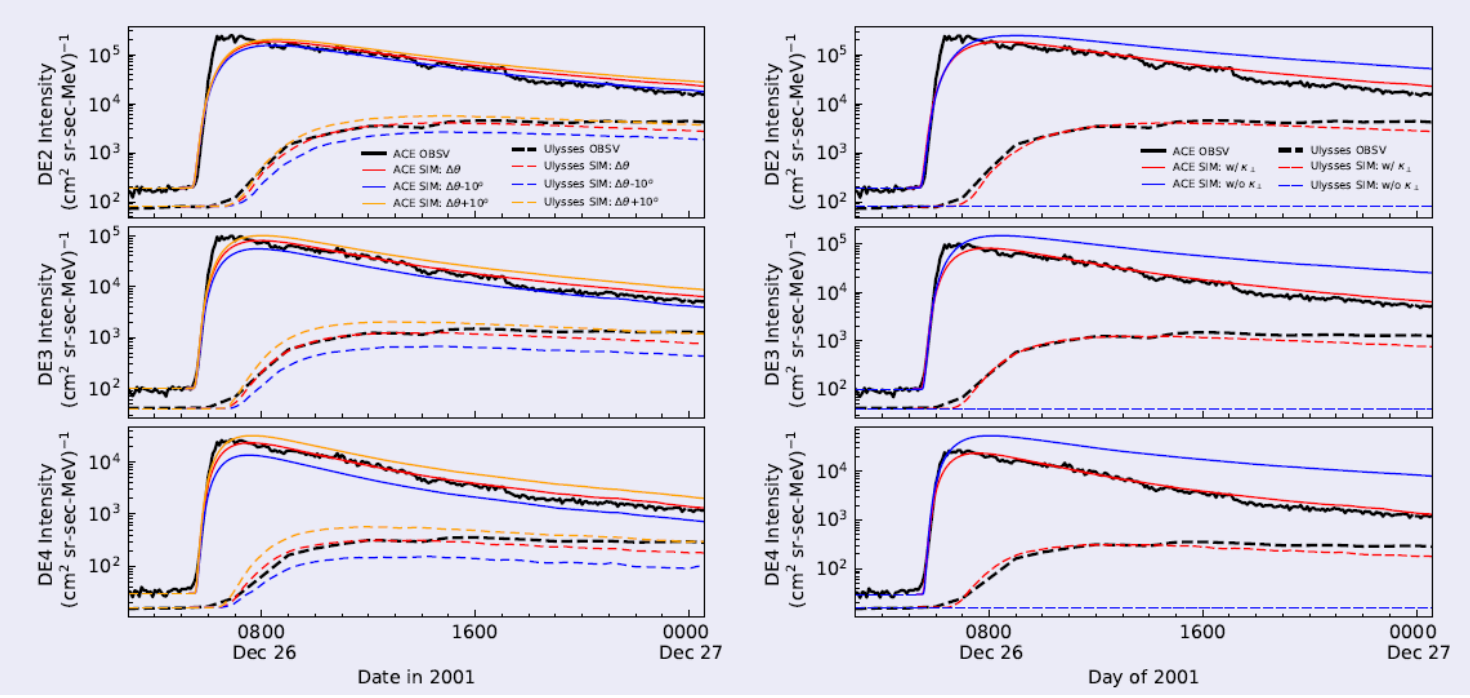

Figure 5: A comparison between SEP observations and model results showing the effect of perpendicular diffusion. Taken from [7].
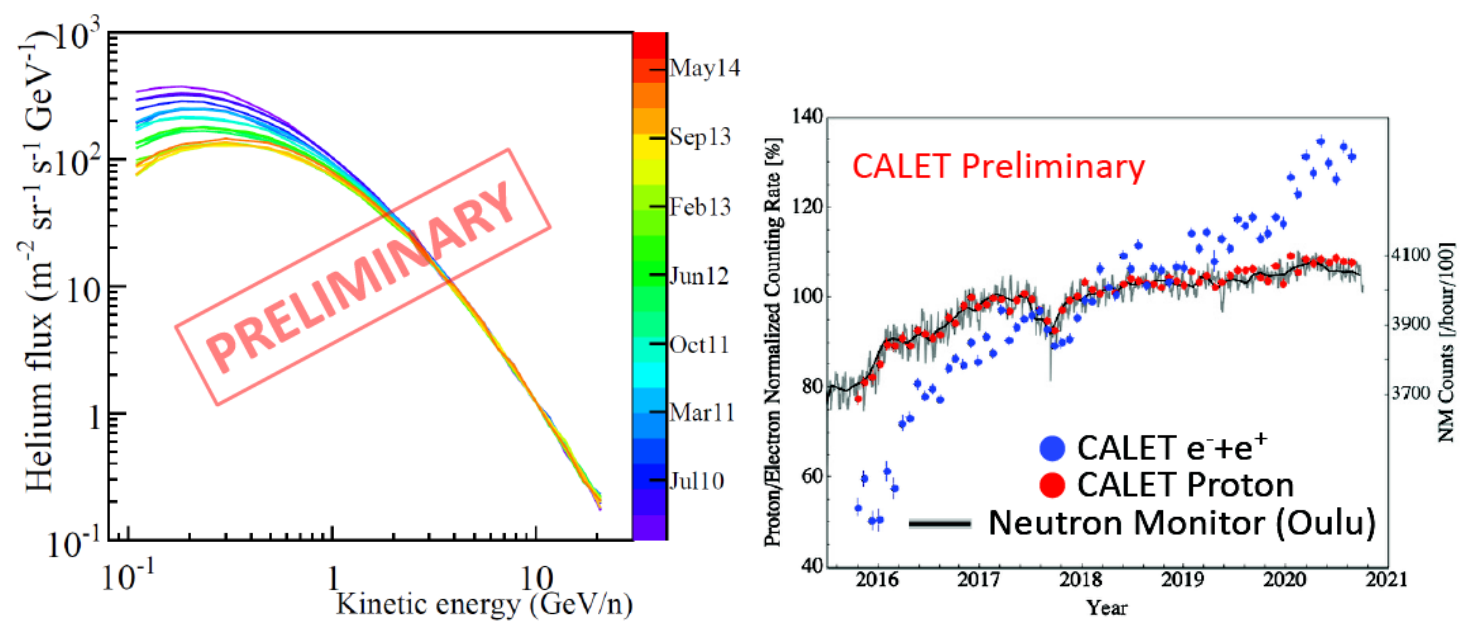

Figure 6: Observations of precision long-term GCR mesurements from [8] and [9].

investigated the effect of turbulent fluctuations on the drift coefficient of SEPs. The perturbed particle motion, characteristic of particles interacting with a turbulent field, is clearly discernable.

An example of SEPs model results being compared to in-situ measurements are shown in Fig. 5. Here the authors show that perpendicular diffusion needs to be included in the SEP model in order to successfully reproduce the observations. While the efficiency (and importance) of perpendicular diffusion as a SEP transport process is still hotly debated, an increasing number of SEP model results indicate that this is indeed an important transport process and must be considered when examining SEP transport. 

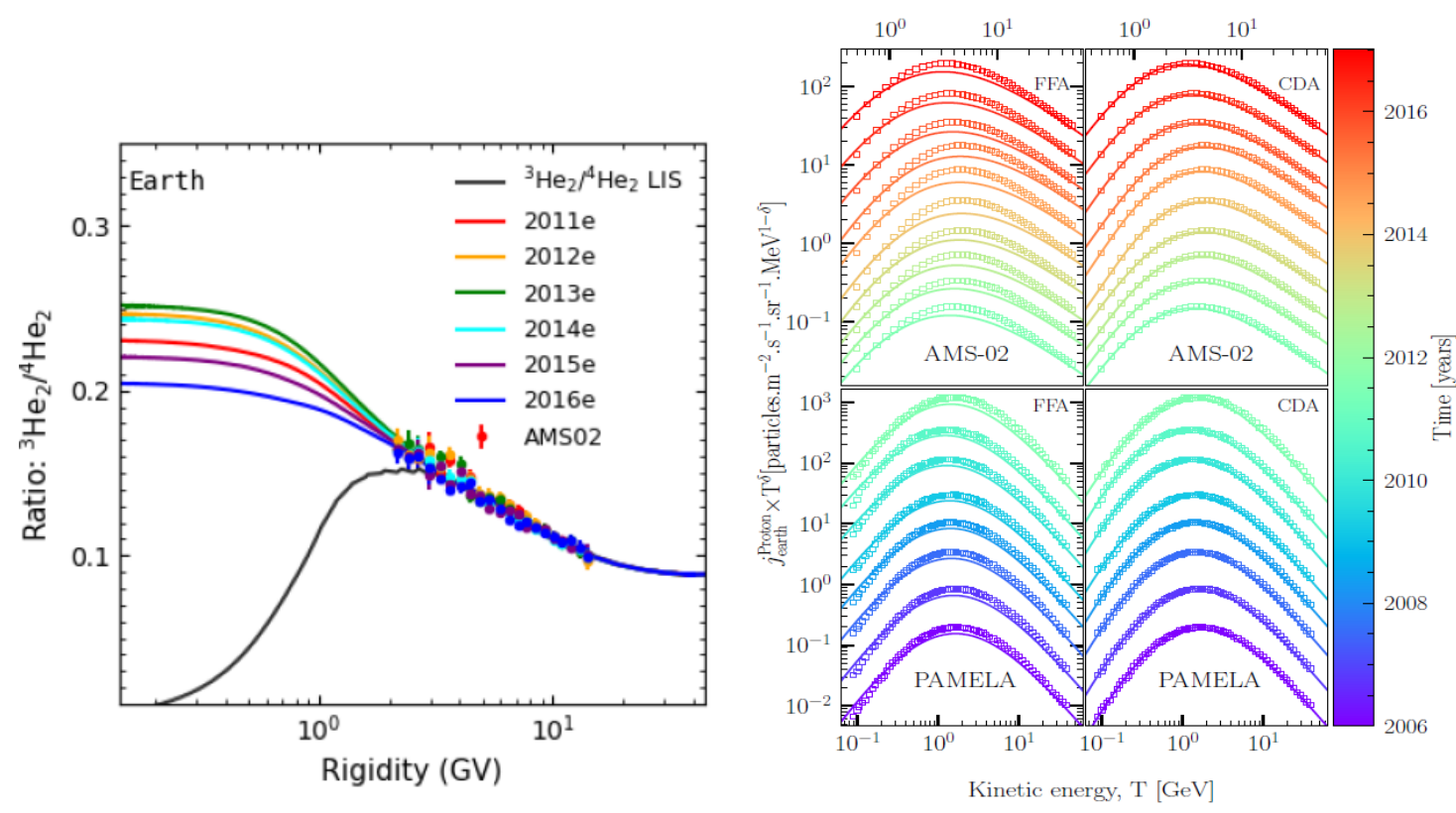

Figure 7: Examples of GCR models with different levels of complexity; the left panel, from [10] shows simulated drift effects of Helium isotopes, while the right panel shows analytical approximations by [11].

\section{Discussion session \#20: GCR long-term modulation}

Fig. 2, and other space-based GCR measurements have shown that the 2020 solar minimum has, once again, been accompanied by record-setting GCR intensities, indicative of very quiet solar conditions. This so-called deep solar minimum is ideal for studying GCR modulation.

Such studies are further facilitated by precision measurements of GCRs by a number of instruments, most notable AMS-02 and PAMELA. As an example, the left panel of Fig. 6 shows very accurate GCR Helium spectra, obtained for a full solar cycle. The right panel of the figure shows a similar example, where we now have access to precision proton and electron time-profiles over (almost) a complete solar cycle. Such charge-sign dependent measurements are ideal for studying drift effects in the heliosphere (differently charged GCRs will drift is opposite directions during the same drift cycle). These measurements will undoubtedly lead to a lot of insight regarding GCR transport in the coming years.

In order to test theories of GCR propagation, GCR measurements are usually compared to model results, where the model solves the relevant GCR transport equation. The complexity of this equation usually necessitates a numerical solution, making such comparisons a cumbersome process. However, based on the physical set-up, several approximations can usually be made to simply the modulation process. In the left panel of Fig. 7, for example, is a comparison between model results and Helium isotope measurements. As yearly measurements are considered, these authors could simplify the modulation process by assuming longitudinal symmetry and solving the model in a simplified 2D spatial geometry. This greatly simplifies the model, while still capturing the most important physical processes, including drift effects. On the other hand, when we're only interested in reproducing observed spectra (e.g. for dosimetric and other space weather purposes), 

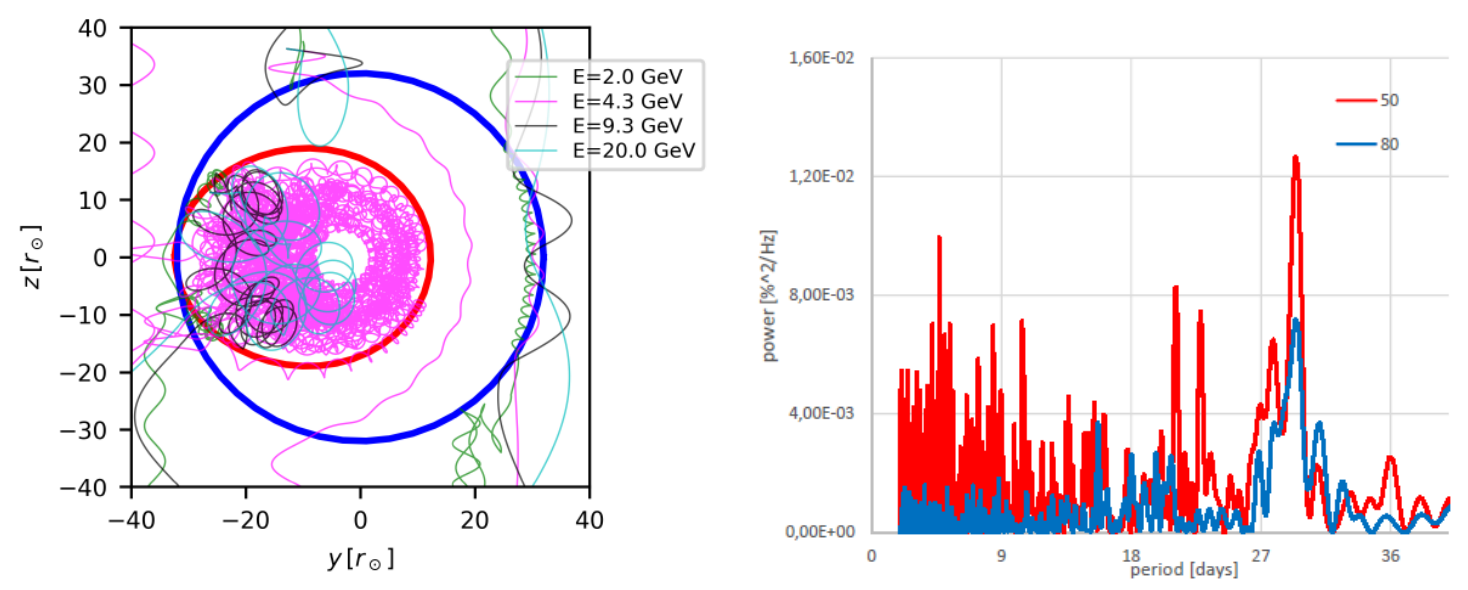

Figure 8: The left panel, from [12], shows full-orbit simulations of GCRs propagating into a magnetic flux rope, embedded inside a CME. The right panel is taken from [13] and shows periodograms for STEREO A proton measurements in the 50 and $80 \mathrm{MeV}$ range. Note the peak near $\sim 27$ days, corresponding to the solar rotation rate.

the modulation process can be reduced even more until analytical approximations are available. This is shown in the right panel of Fig. 7 where the convection-diffusion approximation is compared to proton spectra. However, we should acknowledge that each level of simplification neglects some physical processes, e.g. the convection-diffusion approximation cannot account for drift effects. Often in GCR modulation studies, emphasis is incorrectly put on only using extremely complicated models where, in fact, we could extract much more physics from using a simpler (perhaps even analytical) approach. This is in line with the well-know adage, often misattributed to Einstein: "Everything should be made as simple as possible, but not simpler".

\section{Discussion session \#21: Short-term modulation}

Short term modulation effects refer to temporal changes on timescales from minutes to months and are, most often, associated with solar transients. The most notable short term modulation effects are GCR decreases associated with the passage of CMEs (the so-called Forbush decreases) as well as $\sim 27$ day recurrent GCR decreases associated with the passage of co-rotating (with the Sun) interaction regions (CIRs). Although both CMEs and CIRs drive shock waves that can accelerate particles, we refer here specifically to the high-energy GCR component that decreases when these structures passes an observer.

In the most basic picture of short term modulation, CIRs and CMEs form GCR diffusion barriers that inhibit the diffusion of GCRs across these structures, leading to a depletion in GCR intensity after the structure passes an observer. However, these structures are much more complex that this simple picture may imply. For instance, Forbush decreases generally show a two-step decrease with the initial decrease related to the turbulent sheath regions that surrounds the central flux-rope region of the CME, which is responsible for the second decrease. [12] simulated the propagation of GCRs entering this embedded flux rope region using a full-orbit simulation approach. They find that particles can efficiently enter the flux rope region at magnetic X-points, while pitch-angle 

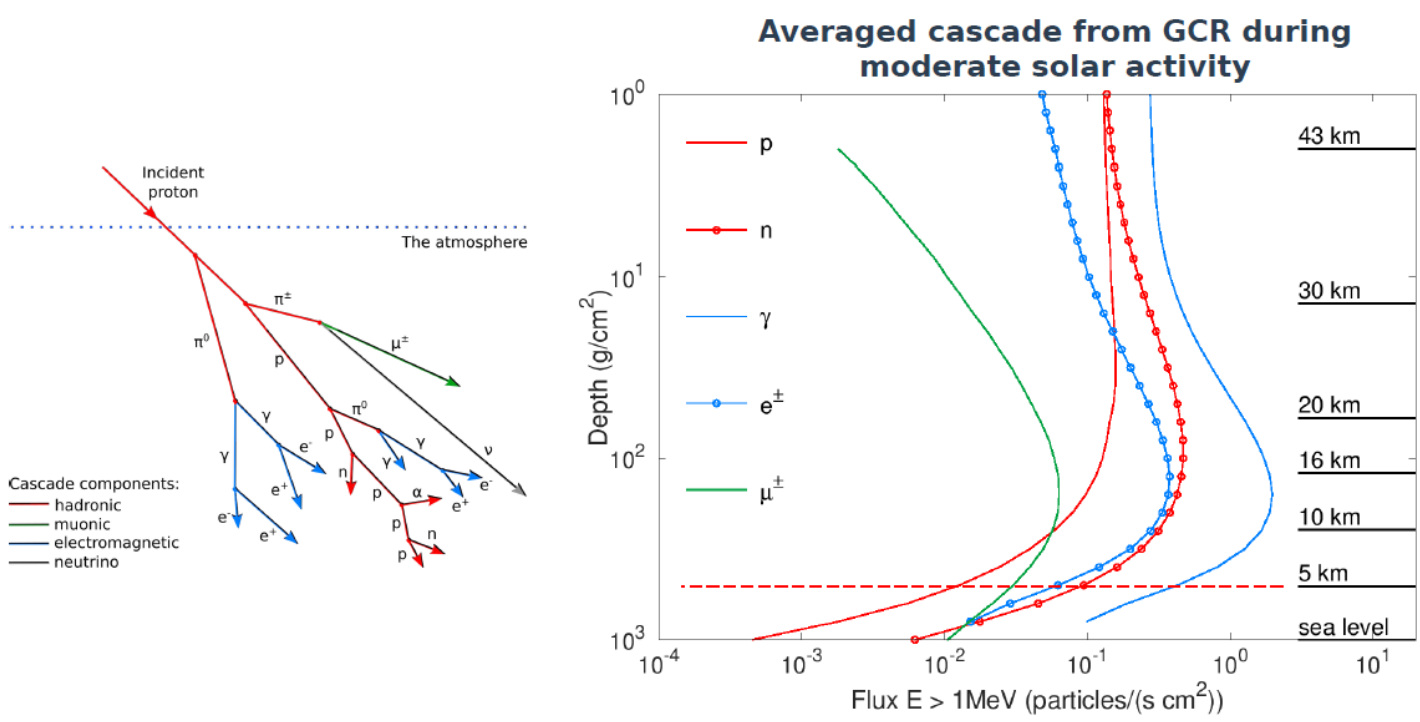

Figure 9: The left panel shows an atmospheric cascade that forms when a high-energy primary CR interacts with atmospheric molecules. The production of these secondary particles are altitude dependent and shown in the right panel. Both figures are taken from [14].

scattering, which then leads to GCRs diffusing towards the center of the flux rope, is a much slower process. The result is that the problem can be modelled as radial diffusion without the need to account for finite Larmor radius effects.

The right panel of Fig. 8 shows results from [13] who studied the energy and polarity dependence of the amplitude of $\sim 27$ day recurrent variations. The authors confirm earlier results that the amplitude of recurrent variations, from neutron monitor (NM) data, is larger in the $A>0$ drift cycle, illustrating that drift effects play a prominent role. However, for low energy space-based observations, no clear polarity dependence is observed. Both the drift cycle dependence, and it's energy dependence, remains to be explained.

\section{Discussion session \#22: Atmospheric effects on CRs}

Neutron monitors (NMs), located on Earth's surface, uses the Earth's geomagnetic field as a spectrometer, and the Earth atmosphere as a particle producer. Before being able to reach Earth's surface, CRs need to enter the geomagnetic field, and need a minimum rigidity (referred to as a cut-off rigidity) to do so. The cut-off rigidity is highly dependent on the geomagnetic field's geometry and is a maximum at the equatorial regions $(\sim 17 \mathrm{GV})$, while decreasing to essentially zero at the polar regions; see e.g. [15]. After entering through the geomagnetic field, CRs can then interact with atmospheric nuclei, forming a cascade of secondary particles (see the left panel of Fig. 9) which may eventually be observed by a NM. In order for secondary particles to be registered on ground level, a primary $\mathrm{CR}$ needs $\sim 1 \mathrm{GV}$ of rigidity to sustain an atmospheric cascade, leading to a so-called atmospheric cut-off. The geomagnetic cut-off dominates the atmospheric cut-off, except at the polar regions where NMs are most sensitive to low energy particles. 

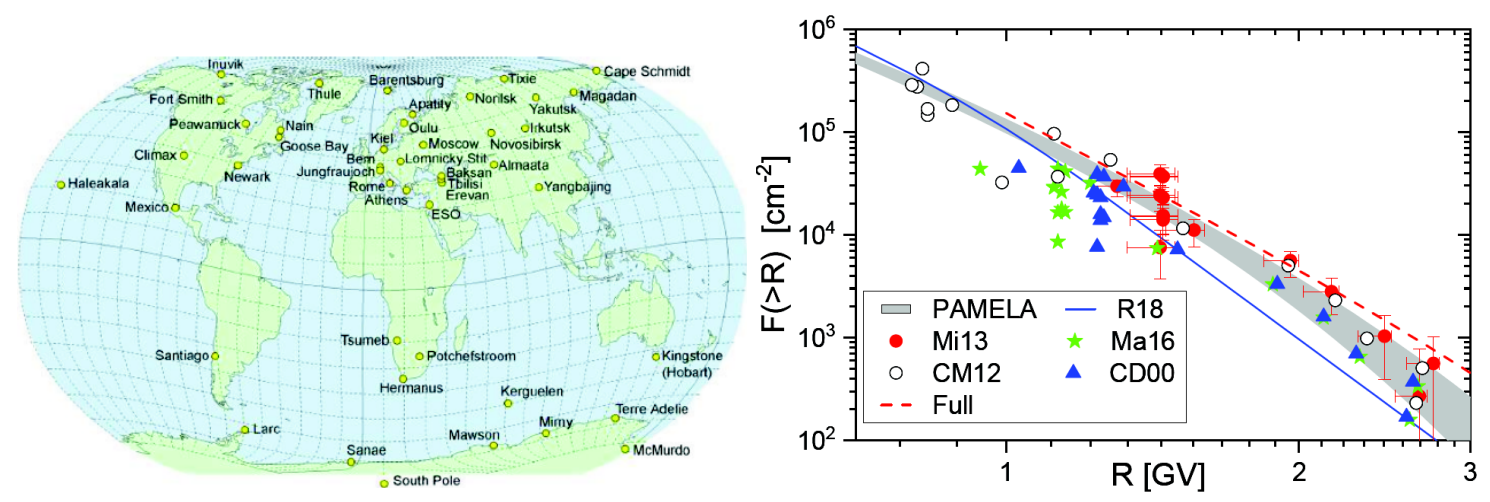

Figure 10: The left panel shows the world-wide network of NM stations that were successfully inter-calibrated by [16] to infer top-of-atmosphere SEP spectra (right panel).

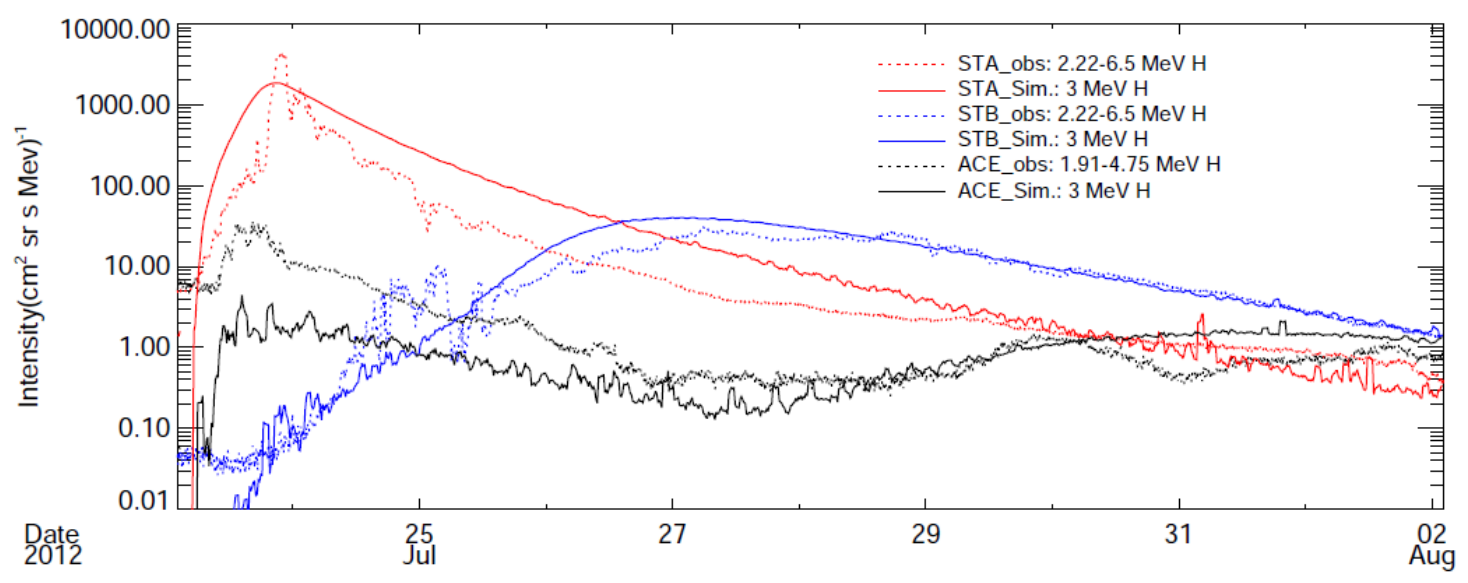

Figure 11: SEP simulation results, from [17], compared to multi-spacecraft SEP observations.

This altitude profile of the atmospheric cut-off is not well studied. The right panel of Fig. 9, taken from [14], shows the that intensity of secondary particles peak near $16 \mathrm{~km}$, decreasing again towards sea-level. It is therefore clear that higher-altitude NMs, at the polar regions, are more sensitive to low energy CR changes. By calculating the altitude dependence of this atmospheric cut-off, [14] successfully explains why high-latitude NMs, at the polar regions, are sensitive to so-called sub-GLEs (sub-ground level enhancements) which are essentially GLEs associated with lower energy $(<1 \mathrm{GV})$ SEP enhancements.

\section{Discussion session \#23: Solar events observed on/near Earth}

The world-wide network of NM stations can, in principle, be used to accurately reconstruct the intensity, spectrum, and directional anisotropy of high-energy SEP events (the so-called GLE events). However, to do so, the different stations, constructed at different times using different designs, must be inter-calibrated as each monitor may therefore have a different efficiency. The so-called yield function, relating the NM count rate at a certain position to the top-of-atmosphere incident particle spectrum, must therefore be determined uniquely for each instrument. [16] presents 

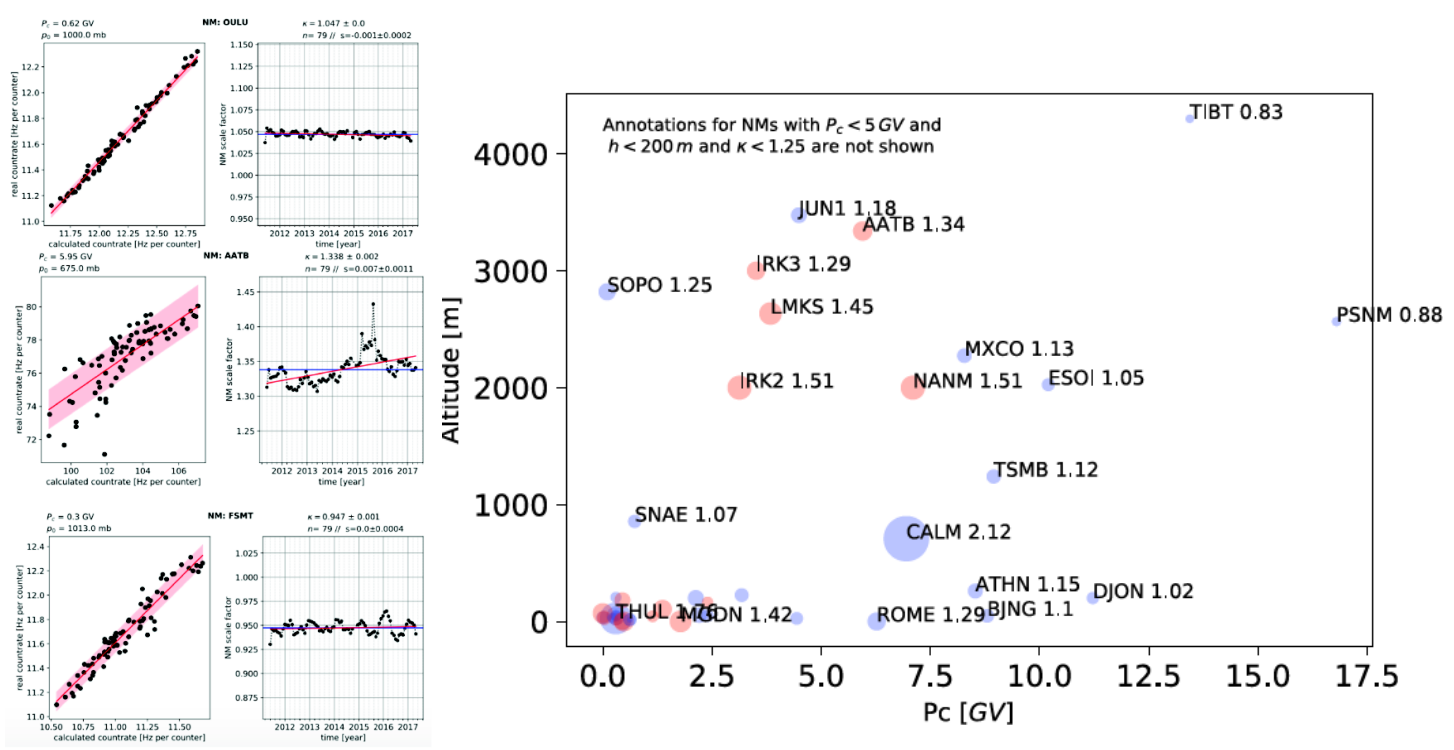

Figure 12: Results from [18] showing the inter-calibration of the world-wide NM network. The right panel shows the derived scaling factor (the size of the symbols corresponds to the magnitude of the factor) that needs to be applied to individual NM stations as a function of cut-off rigidity and altitude.

results from such GLE reconstructions, with results from that study presented in Fig. 10. Newer data analysis techniques make this possible, together with high precision spectra measured by e.g. PAMELA and AMS-02, allowing for an accurate and direct determination of the yield function. By using the technique presented by e.g. [16] we would be able to also invert historical GLE observations in order to study the spectra of historical SEP events which present a very interesting prospect.

Once SEPs are accelerated at/near the Sun, these particles still need to travel through the turbulent interplanetary magnetic field to reach an observer at e.g. Earth. While pitch-angle scattering, leading to field-aligned transport, is relatively well understood, scattering of SEPs perpendicular to the mean field (perpendicular or cross-field diffusion) is not. In order to study the (possibly significant) effect of perpendicular diffusion on SEP intensities, so-called wide-spread SEP events are studied, where the broad longitudinal distribution of the SEPs can be measured by multiple spacecraft, sometimes significantly separated in longitude. An example of such simulations are presented in Fig. 11 where SEP simulation results from [17] are compared to multi-spacecraft observations. These authors show that they are able to reproduce the SEP events fairly well when perpendicular diffusion is included in their model. This indicates that perpendicular diffusion is an important SEP transport mechanism and should be considered when studying SEP events.

\section{Discussion session \#24: Ground-based measurements of low-energy GCRs}

In the previous section it was already mentioned that the world-wide NM network needs to be inter-calibrated before top-of-atmosphere spectra can be obtained. Further details of this calibration process is presented by [18]. Here the authors also considered the newly developed altitude-dependent NM yield functions, and calibrate these to in-situ AMS-02 top-of-atmosphere 

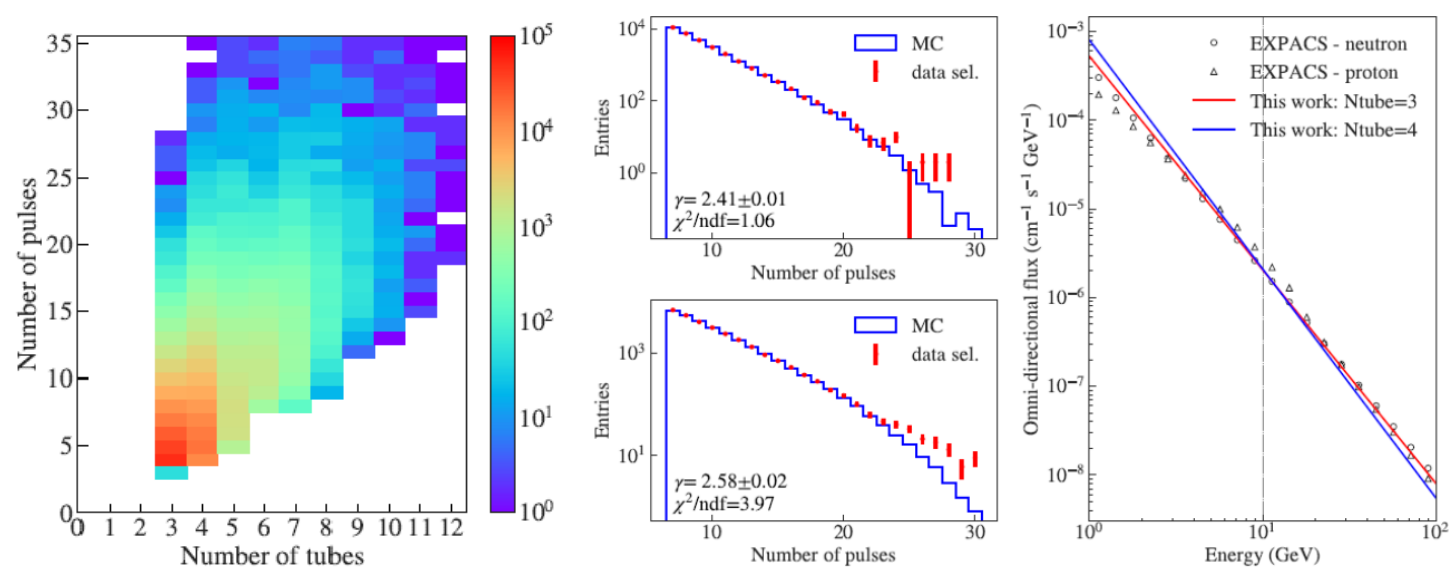

Figure 13: Results from [19] showing how the distribution of neutrons inside a NM can be used to infer the spectrum of the incident secondary particles.
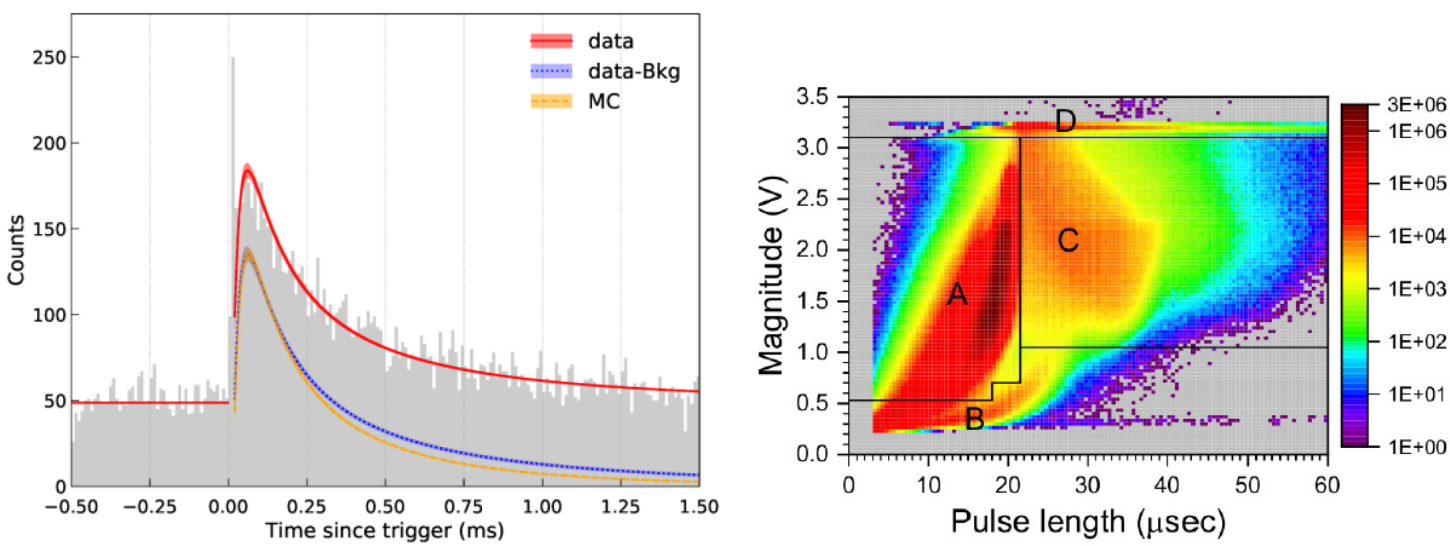

Figure 14: The left panel shows the neutron time distribution measured inside a NM, from [20], along with results of a diffusion-absorption model. The right panel shows the different neutron populations, identified by [21], inside a mini-NM.

spectra. They find a so-called scaling factor for each NM station that can be used for the intercalibration, with these factors shown in Fig. 12. Having such verified scaling factors will allow the global NM network to operate as an efficient spectrometer.

In principle it is also possible to constrain the spectrum of the secondary particles incident on a NM by examining the time and spatial structure of neutrons produced inside the monitor itself, making the inter-calibration of multiple NM stations superfluous. Such an approach was presented by [19], with their results shown in Fig. 13. Here they compare measurements and simulations of particle beams incident on the Princess Sirindhorn NM on Doi Inthanon, located in Thailand. This technique, showing a lot of promise, if currently being studied in more detail.

\section{Selected presentations from the Presenter's Forum}

Many of discussion sessions touched on the use of NM data and how new technologies and techniques can be used to extract additional information from these old instruments. Results from 


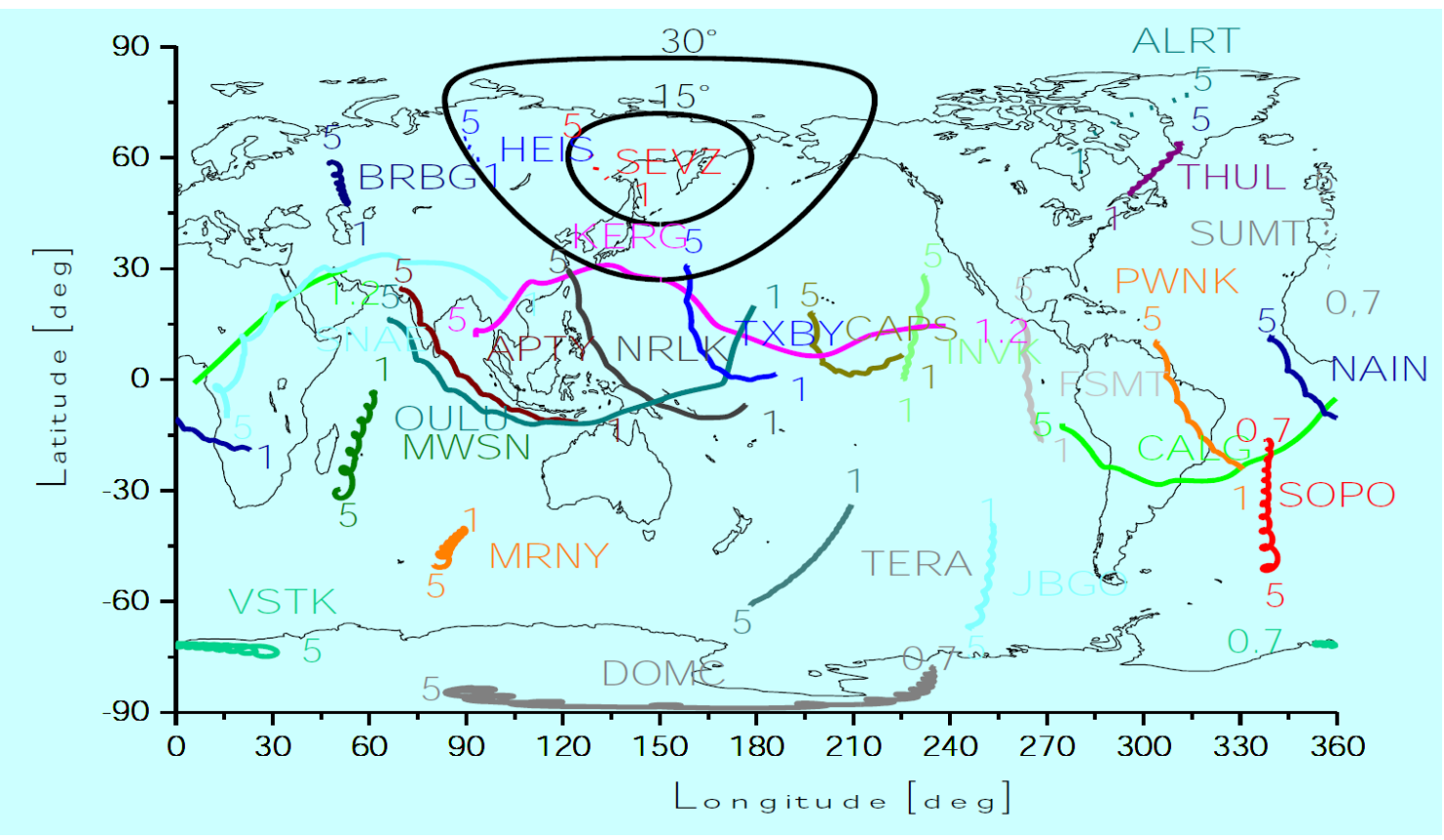

Figure 15: Results from [22], showing the current NM stations, along with the cone-of-acceptance (coloured lines) at different rigidities for different NM stations. The authors identify a gap in the current NM network, illustrated here by the circles over eastern Russia.

[20] and [21], for example, illustrate how the use of new, and much faster, digitization systems can be used to measure and/or infer the distribution of neutrons inside the detector. The left panel of Fig. 14, from [20], shows the temporal distribution of neutrons measured by a single tube of a larger detector. These are the first such direct measurements and can be understood through the production, absorption, and diffusion of neutrons inside the NM. The right panel of the figure, from [21], shows the distribution of the pulses, in terms of length and amplitude, measured inside a mini-NM. These authors identify different neutron populations, including (possibly) a minor muon component.

As SEP events, and therefore also GLE events, are highly anisotropic, a number of NM stations, distributed over the Earth's surface is needed to capture all the details of a GLE event. The inversion techniques discussed in this summary therefore relies on good spatial coverage of the world-wide NM network as each monitor is only sensitive to particles reaching it from very specific regions, i.e. it's own cone-of-acceptance. This coverage was studied by [22]. These authors also discuss how this network can be optimised to be more sensitive to GLE events with the construction of four new stations, and by re-opening four older decommissioned sites. Interestingly, they also identify gaps in the current network, with an example shown in Fig. 15. More importantly, these authors emphasise the need to continue funding and operating the current network of monitors.

\section{Summary}

Hopefully, by means of this summary paper, I could give the reader a glimpse into the rich and varied field of cosmic ray acceleration and transport in the heliosphere. Many of these topics were 
expertly addressed by presenters during the 2021 ICRC and I could only summarize a very small fraction of the excellent work currently being done in the field. Together with recent advances in theory and simulations, the abundance of in-situ CR measurements both inside and outside of the heliosphere has really given rise to the golden age of heliophysics.

\section{References}

[1] R.D. Strauss \& M.S. Potgieter, Is the Highest Cosmic-Ray Flux Yet to Come?, Sol. Phys. 289 (3197) 2014.

[2] S. Fu, X. Zhang, L.-L. Zhao, \& Y. Li, Variations of the Galactic Cosmic Rays in the Recent Solar Cycles, ApJS 254 (37) 2021.

[3] R.A. Mewaldt, Galactic Cosmic-Ray Intensities During three Solar Minima, this conference, [https://pos.sissa.it/395/1302].

[4] C. Cohen, et al., Parker Solar Probe's Measurements of the 29 November 2020 Solar Energetic Particle Event, this conference, [https://doi.org/10.22323/1.395.1292].

[5] F.J. Effenbeger, Anomalous Transport and Acceleration of Energetic Particles, this conference, [https://pos.sissa.it/395/1340].

[6] J.P. van den Berg, Turbulent Reduction of Drifts for Solar Energetic Particles, this conference, [https://pos.sissa.it/395/1339].

[7] L.L. Lian et al., Observations and numerical simulations of gradual SEP events with Ulysses and ACE, this conference, [https://doi .org/10.22323/1.395.1351].

[8] N. Marcelli et al., A full solar cycle of proton and helium measurements, this conference, [https://doi.org/10.22323/1.395.1283].

[9] S. Miyake et al., Solar Modulation During the Descending Phase of Solar Cycle 24 Observed with CALET on the International Space Station, this conference, [https://doi.org/10.22323/1.395.1270].

[10] M.D. Ngobeni et al., Numerical modeling of the solar modulation of helium isotopes in the inner heliosphere, this conference, [https://doi.org/10.22323/1.395.1338].

[11] M.G. Mosotho, Spectral parameterization of GCR observations and reconstruction of solar modulation parameters derived from the Convection-Diffusion approximation, this conference, [https://pos.sissa.it/395/1269].

[12] T. Laitinen \& S. Dalla, Access of cosmic rays to an ICME from external field lines, this conference, [https://doi.org/10.22323/1.395.1359].

[13] R. Modzelewska \& A. Gil, Periodic variations of GCR intensity and anisotropy related to solar rotation by ACE/CRIS, STEREO, SOHO/EPHIN and neutron monitors observations, this conference, [https://doi.org/10.22323/1.395.1271]. 
[14] S. Poluianov \& A. Mishev, The altitude profile of the cosmic ray atmospheric cutoff, this conference, [https://doi.org/10.22323/1.395.1331].

[15] D. Gecáŝek et al., Transparency of magnetosphere for cosmic rays in last two millennia, this conference, [https://doi .org/10.22323/1.395.1335].

[16] S. Koldobskiy et al., New reconstruction of the event-integrated spectra for GLE events, this conference, [https://doi.org/10.22323/1.395.1273].

[17] S. Qi \& G. Qin, The 23 July 2012 SEP event numerical simulation with multi-spacecraft observation data, this conference, [https://doi .org/10.22323/1.395.1347].

[18] A. Mishev et al., New neutron monitor altitude-dependent yield function and its application to an analysis of neutron-monitor data, this conference, [https://doi.org/10.22323/1.395.1247].

[19] P. Evenson et al., Multiple Particle Detection in a Neutron Monitor, this conference, [https://doi.org/10.22323/1.395.1240].

[20] K. Chaiwongkhot et al., Measurement and Simulation of the Neutron Travel Time Distribution inside a Neutron Monitor, this conference, [https://doi .org/10.22323/1.395.1277].

[21] M. Similä et al., Pulse height-length analysis of data from neutron monitors DOMC/DOMB with a new data acquisition system, this conference, [https://doi.org/10.22323/1.395.1237].

[22] A. Mishev \& I. Usoskin, Performance of the current and extended global NM network for solar particle registration and analysis, this conference, [https://doi.org/10.22323/1.395.1315]. 